EDITORIAL

\title{
Helping the FTD Patient-Caregiver Dyad
}

Can. J. Neurol. Sci. 2011; 38: 671-672

"To cure sometimes, to relieve often, to comfort always."

- Edward Livingston Trudeau

Like Alzheimer's disease (AD), Picks disease, or its contemporary incarnation Frontotemporal dementia (FTD) ${ }^{1}$, is a clinical neurodegenerative syndrome of insidious onset that ultimately strips the afflicted of all cognitive and functional independence and inexorably leads to death, usually within a decade of initial detectable changes. Although less common across age groups, it's prevalence is equal to that of $\mathrm{AD}$ in individuals under that age of 65 , with onset usually between 45 and 65 years $^{2,3}$.

Despite preceding the description of AD by nearly a decade, FTD has remained in the shadows of its younger cousin, both from a clinical and a scientific point of view. But, academic interest in FTD is growing, as reflected by the number of PubMed publications on the topic. Peaks in publication occurred following consensus meetings in $1994^{4}$ and $1998^{5}$, and more recently following the identification of the elusive ubiquitinated protein inclusions TDP- $43^{6,7}$ and FUS ${ }^{8}$, as well as the role of progranulin mutations ${ }^{9,10}$. In fact, our exploding understanding of the molecular neuropathological underpinnings and their correlation to the clinical manifestations of this heterogeneous group of disorders is also leading to the isolation of more pathologically homogeneous groups during life $\mathrm{e}^{11}$ and the appearance of clinical trials designed to address etiology ${ }^{12}$. Although current therapies are limited to symptomatic palliation $^{13}$, successful disease modifying treatment will likely emerge within our lifetimes.

Patients with FTD have also contributed selflessly to the advancement of our understanding of brain-behavior correlations: Application of modern brain imaging techniques to these patients has uncovered the neuroanatomical substrates of complex concepts such as empathy, recognition of emotions and emotional blunting ${ }^{14}$, aberrant motor behavior and stereotypies ${ }^{15}$, apathy $^{15}$, disinhibition ${ }^{15}$, binge eating ${ }^{16}$, self appraisal ${ }^{17}$, and even interpersonal traits ${ }^{18}$. Cognitive and behavioral neuroscience has made giant strides in our understanding of what is it to be human thanks to the study of patients with this disorder.

But what are we doing for these patients now? What do these patients and their caregivers actually need the most? Frontotemporal dementia strikes in the prime of life. Patients are often fathers or mothers of young families and in the midst of their professional careers. The social, professional, and even legal consequences of the disorder are frequently devastating and, unlike those of the later onset Alzheimer's disease, have an impact that reaches far beyond the individual patient.

As is often the case with chronic diseases, patients and especially for dementias - caregivers will be the impetus for the creation of support groups and ultimately associations like the Alzheimer's Society, and for FTD, the Association for the FTDs (www.theaftd.org), created in 2002. These groups, and numerous other players involved in the diagnosis, management, and study of patients with FTD address ongoing issues that occur once diagnosis has been made.

In their paper in this edition of the CJNS ${ }^{19}$, Tiffany Chow and her colleagues from the University of Toronto and Dalhousie University, have basically asked their patients' caregivers "how are we doing?" Their questions were simple (and paraphrased here): In addition to probing for the initial manifestations of the disease with "What were the first signs of disease?", they explored the impact of the disease and of the diagnostic process on their lives with "What frustrates or frustrated you most?", "Who or what helped you most?", and "What surprised you the most?" Through an innovative mechanism using an online survey technique, they were able to obtain candid opinions and responses to their questions from caregivers from Canada, the US, Britain, and Europe. With all the caveats associated with voluntary online surveys, the results of their study are rather telling.

Concerning initial changes, the most frequent was "change in thinking and judgment", which included "insensitivity". The most troublesome aspects of caregiving included "poor insight", "caregiver quality of life". What surprised caregivers the most was that "dementia can affect young people". Finally, resources or interventions that help the most are striking: "the treating neurologist" came first, followed by "the Internet".

In isolating the treating neurologist and the Internet as the two most helpful resources, their data betrays the prolonged frustration experienced by the caregivers in getting the correct diagnosis and the appropriate guidance and help. The problem is, FTD is hard to recognize early, and hard to diagnose. Families are aware that something is awry long, long before a correct diagnosis is suggested. In contrast to $\mathrm{AD}$, where the lead-time to diagnosis can be up to three years after appearance of noticeable changes, patients with FTD can progress for five or more years before diagnostic efforts begin to focus on this etiology. Although caregivers of patients with FTD may wait twice as long as caregivers of patients with AD before seeking medical advice, physicians will take nearly an extra year to actually make the diagnosis. In addition, twice as many patients with FTD are likely to receive initial non-dementia diagnoses, including psychiatric conditions ${ }^{20}$.

Indeed, if we are going to help the caregivers and this patient population (and in particular if we hope to get them involved in early etiological treatment trials), we need to educate the public about the existence of "dementia in the not-so-old". Further, in an effort to shorten the delay in seeking medical advice, families need to understand and recognize the earliest changes associated with this group of disorders ${ }^{21}$. This study clearly identifies the Internet as a resource for supplying appropriate information to those unwitting caregivers reaching out and grasping to understand what is happening to their loved one. 
Any delay to seeking medical advice notwithstanding, the onus really falls upon us to educate ourselves. We need to be able to make the diagnosis earlier and refer to specialized tertiary care centers where appropriate interventions such as clinical trials, specialized daycare, and disease-adapted legal counseling can be obtained $^{22-24}$. Because studies such as this one also capture the caregivers' first appreciation of change, they help to translate caregiver complaints into a differential diagnosis that must include FTD.

Clearly, further studies of this kind will help bridge the gap between what both families and doctors know and what we need to know, not only to better comfort, but, ultimately, to cure this devastating disorder.

Gabriel C. Léger, Fadi Massoud Centre Hospitalier de l'Université de Montréal (CHUM) Montreal, Quebec, Canada

\section{REFERENCES}

1. Piguet $\mathrm{O}$, Hornberger $\mathrm{M}$, Mioshi $\mathrm{E}$, et al. Behavioural-variant frontotemporal dementia: diagnosis, clinical staging, and management. Lancet Neurol. 2011a;10(2):162-72.

2. Knopman D, Petersen R, Edland S, et al. The incidence of frontotemporal lobar degeneration in Rochester, Minnesota, 1990 through 1994. Neurology. 2004;62:506-8.

3. Ratnavalli E, Brayne $\mathrm{C}$, Dawson $\mathrm{K}$, et al. The prevalence of frontotemporal dementia. Neurology. 2002;58:1615-21.

4. Clinical and neuropathological criteria for frontotemporal dementia. The Lund and Manchester Groups. J Neurol Neurosurg Psychiatry. 1994;57:416-18.

5. Neary D, Snowden J, Gustafson L, et al. Frontotemporal lobar degeneration: a consensus on clinical diagnostic criteria. Neurology. 1998;51:1546-54.

6. Arai T, Hasegawa M, Akiyama $\mathrm{H}$, et al. TDP-43 is a component of ubiquitin-positive tau-negative inclusions in frontotemporal lobar degeneration and amyotrophic lateral sclerosis. Biochem Biophys Res Commun. 2006;351:602-11.

7. Neumann M, Sampathu D, Kwong L, et al. Ubiquitinated TDP-43 in frontotemporal lobar degeneration and amyotrophic lateral sclerosis. Science. 2006;314:130-3.

8. Neumann M, Rademakers R, Roeber S, et al. A new subtype of frontotemporal lobar degeneration with FUS pathology. Brain. 2009;132:2922-31.

9. Baker M, Mackenzie I, Pickering-Brown S, et al. Mutations in progranulin cause tau-negative frontotemporal dementia linked to chromosome 17. Nature. 2006;442:916-19.

10. Cruts $\mathbf{M}$, Gijselinck $\mathbf{I}$, van der Zee J, et al. Null mutations in progranulin cause ubiquitin-positive frontotemporal dementia linked to chromosome 17q21. Nature. 2006;442:920-4.

11. Boeve BF. Links between frontotemporal lobar degeneration, corticobasal degeneration, progressive supranuclear palsy, and amyotrophic lateral sclerosis. Alzheimer Dis Assoc Disord. 2007;21:S31-8.

12. Gozes I. Tau pathology and future therapeutics. Curr Alzheimer Res. 2010;7:685-96.

13. Kaye E, Petrovic-Poljak A, Verhoeff N, et al. Frontotemporal dementia and pharmacologic interventions. J Neuropsychiatry Clin Neurosci. 2010;22(1):19-29.

14. Rosen HJ, Wilson MR, Schauer GF, et al. Neuroanatomical correlates of impaired recognition of emotion in dementia. Neuropsychologia. 2006;44:365-73.

15. Rosen HJ, Allison SC, Schauer GF, et al. Neuroanatomical correlates of behavioural disorders in dementia. Brain. 2005;128: 2612-25.
16. Woolley JD, Gorno-Tempini ML, Seeley WW, et al. Binge eating is associated with right orbitofrontal-insular-striatal atrophy in frontotemporal dementia. Neurology. 2007;69:1424-33.

17. Rosen HJ, Alcantar $\mathbf{O}$, Rothlind $\mathbf{J}$, et al. Neuroanatomical correlates of cognitive self-appraisal in neurodegenerative disease. NeuroImage. 2010;49:3358-64.

18. Sollberger M, Stanley CM, Wilson SM, et al. Neural basis of interpersonal traits in neurodegenerative diseases. Neuropsychologia. 2009;47:2812-27.

19. Chow TW, Pio FJ, Rockwood K. An international needs assessment of caregivers for frontotemporal dementia. Can J Neurol Sci. 2011;38(5):753-7.

20. Rosness TA, Haugen PK, Passant U, et al. Frontotemporal dementia: a clinically complex diagnosis. Int $\mathbf{J}$ Geriatr Psychiatry. 2008;23:837-42.

21. Hallam B, Silverberg N, Lamarre A, et al. Clinical presentation of prodromal frontotemporal dementia. Am J Alzheimers Dis Other Demen. 2007;22:456-67.

22. Banks S, Rogalski E, Medina $J$, et al. Organizing a series of education and support conferences for caregivers of individuals with frontotemporal dementia and primary progressive aphasia. Alzheimers Care Q. 2006;7:243-50.

23. Grinberg A, Lagunoff $\mathbf{J}$, Phillips $\mathbf{D}$, et al. Multidisciplinary design and implementation of a day program specialized for the frontotemporal dementias. Am J Alzheimers Dis Other Demen. 2007;22:499-506.

24. Rosness TA, Haugen PK, Engedal K. Support to family carers of patients with frontotemporal dementia. Aging Ment Health. $2008 ; 12: 462-6$. 